*Preprint*

\title{
No effect of cycling shoe sole stiffness on sprint performance
}

James W. Hurt III ${ }^{1 *}$ and Rodger $\mathrm{Kram}^{2}$

1. Department of Mechanical Engineering, University of Colorado, Boulder, U.S.A.

2. Department of Integrative Physiology, University of Colorado, Boulder, U.S.A.

*Corresponding author. Email: tripphurt@gmail.com.

Author agreement statement: We the authors agree to the sharing of this preprint on SportR $\chi$ iv.

Twitter handles: @TrippFallHurt |@RodgerKram

DOI

https://doi.org/10.31236/osf.io/k6bh8

\section{Citation}

Hurt, J. W., III, \& Kram, R. (n.d.). No effect of cycling shoe sole stiffness on sprint performance. Retrieved from osf.io/preprints/sportrxiv/k6bh8 


\title{
No effect of cycling shoe sole stiffness on sprint performance
}

\begin{abstract}
Most competitive and recreational road cyclists use stiff-soled shoes designed for cycling and "clipless" pedals that firmly attach to the shoes. There are many unsubstantiated claims by cyclists and industry professionals about the advantages of cycling shoes and clipless pedals. Scientific research has shown that cycling shoes and clipless pedals have no significant effects on the metabolic cost of cycling during submaximal, steady-state efforts. However, a recent study demonstrated that, compared to running shoes, cycling shoes and clipless pedals do provide performance benefits relevant to sprint cycling. Here, we investigated if there was a positive relationship between longitudinal bending stiffness of cycling shoe soles and sprint performance. We measured the mechanical power outputs, velocities, and cadences of 19 healthy male recreational/competitive cyclists during maximal sprint cycling. Participants rode outdoors on a paved asphalt road with a steady, uphill grade of $4.9 \%$. Each subject completed nine $50 \mathrm{~m}$ cycling sprints in three (single-blinded) shoe conditions: identical shoe uppers with injection moulded nylon soles, carbon fibre-fibreglass blend soles, and full carbon fibre soles. The same clipless pedals were used throughout all tests. No significant differences were detected between the three shoe soles for: $50 \mathrm{~m}$ average and peak 1-second power, average change and peak change in velocity, average and peak cadence, maximal sprint velocity, peak acceleration, and peak crank torque (all $\mathrm{p}>0.31$ ). Greater longitudinal bending stiffness of cycling shoe soles had no effect on sprint performance during short uphill sprints.
\end{abstract}

Keywords: biomechanics; footwear; bicycling; power Word Count: Abstract: 240, Main Text: 3556

\section{Introduction}

Most competitive and recreational road cyclists use stiff-soled cycling shoes and "clipless" pedals, which firmly attach the shoe to the pedal. There are many claims that cycling shoes, shoe 
insoles, and shoe-pedal interfaces improve efficiency. However, for aerobic, low intensity cycling, at least three studies have shown that there are no differences in cycling metabolic efficiency between three pedal interfaces -flat pedals, toe-clip pedals, and modern clipless pedals (Morneiux et al., 2008; Ostler et al., 2008; Straw and Kram, 2016). Additionally, foot orthoses and cycling shoe insoles have no effect on cycling metabolic efficiency in healthy individuals (Anderson and Sockler, 1990). Similarly, flexible running shoes are equally efficient as rigidsoled cycling shoes during low-intensity (150 W) cycling (Straw and Kram, 2016). Why do cyclists choose to use stiff-soled shoes and clipless pedals?

Cyclists commonly state that they prefer cycling shoes and clipless pedals because they facilitate pulling up on the pedals during the upstroke. Morneiux et al. (2008) investigated this idea by focusing on the pulling-up action during aerobic, low intensity cycling. They found that, when prompted with visual feedback, cyclists were able to increase their pedalling effectiveness, defined as the fraction of the linear impulse applied to the pedal that generates angular crank impulse, at three different points in the crank cycle. However, intentionally pulling up on the pedals had a detrimental effect on metabolic efficiency.

Perhaps cyclists prefer to use stiff-soled cycling shoes with clipless pedals because together they enhance sprint performance. Koch et al. (2013) investigated the effect of cyclingspecific carbon fibre insoles during indoor Wingate sprints. Each rider used their own cycling shoes and pedal system but with two insole conditions: a placebo insole made of standard foam rubber and a cycling specific carbon fibre insole. The subjects were blinded to the insoles. The study found no significant differences between the standard insoles and the cycling specific carbon fibre insoles with regards to peak power or average power during a Wingate anaerobic power test. More recently, Burns and Kram (in press) reported that stiff-soled cycling shoes and 
clipless pedals have dramatic positive effects on several indicators of sprinting performance versus flexible running shoes with toe-clip pedals.

Our primary goal was to investigate if cycling shoe sole stiffness affects sprint cycling performance. We quantified the mechanical power output, velocities, and cadences of riders during a series of uphill sprints. We compared three different commercially available cycling shoe soles that varied in longitudinal bending stiffness. Our overall hypothesis was that the longitudinally stiffest cycling shoe soles would yield the greatest performance during the sprints, and that the least stiff cycling shoe soles would yield the worst performance metrics. We tested the null hypothesis that there would be no statistical differences in performance between the cycling shoe soles during sprinting.

\section{Methods}

Nineteen male recreational/competitive cyclists volunteered and completed the study $(30.2 \pm 7.7$ years old (mean $\pm \mathrm{SD}$ ), body mass of $73.8 \pm 7.2 \mathrm{~kg}$, height $179.0 \pm 3.4 \mathrm{~cm})$. They self-reported a minimum of four hours of cycling per week, averaging $9.2 \pm 3.2$ hours per week and $10.5 \pm 6.9$ years of road cycling experience. Inclusion criteria were as follows: at least 18 years old, in good health, wear European size 43 or 44 shoes (to fit in the available testing shoes), and be able to comfortably ride the $56 \mathrm{~cm}$ frame size testing bicycle. Due to the size requirements of the shoes and the testing bicycle, all subjects who volunteered were male. All subjects gave written informed consent per the University of Colorado Institution Review board.

All subjects rode the same road bicycle (Specialized Roubaix, Morgan Hill, CA, U.S.A.) equipped with a crank-based mechanical power meter (Quarq, Spearfish, SD, U.S.A.). The cranks were $172.5 \mathrm{~mm}$ long and the tyres were inflated to a pressure of 90 pounds per square 
inch before each subject was tested. We locked out the FutureShock® front suspension that was included on the testing bicycle. The testing bicycle also included a crank-mounted cadence sensor and a rear-hub-mounted velocity sensor, both manufactured by Garmin. A Garmin 1000 head unit (Olathe, KA, U.S.A.) mounted to the stem of the testing bicycle recorded the power, velocity, and cadence data. Riders used the same clipless pedals during each test (Shimano PDR7000 105 SPD-SL). To minimize any temperature effects on the strain gauges of the crankbased power meter, the bicycle acclimated to the outdoor environment for 30 minutes prior to testing. We calibrated (zero-ed) the power meter with the right side crank in the 6 o'clock position with no load just before and after testing. There was an average of $8 \pm 6$ "points" difference in calibration factor. For a Quarq power meter, 8 offset points is equal to $0.25 \mathrm{~N}-\mathrm{m}$ of torque which equates to 2-3 $\mathrm{W}$ at cadences of 80-100 RPM. The manufacturer recommends that the offset points should not drift more than 50 points from the start to the end of the ride for optimal accuracy.

After initial screening, subjects reported to the outdoor testing site, a low-traffic, residential street with a steady uphill grade of $4.9 \%$ (2.8 degrees), and smooth and clean asphalt that is largely sheltered from the wind by houses and trees. We measured a $50 \mathrm{~m}$ course with a surveyor's wheel and marked it on the pavement. We studied outdoor sprinting because indoor ergometer sprinting is less ecologically relevant. Uphill sprinting slowed the velocities of the riders and was thus, in the case of an accident, safer than level sprinting. Uphill sprinting from a rolling start is highly relevant to racing where critical accelerations are often made during long climbs in the mountains.

Subjects warmed up ad libitum by riding the testing bicycle on the local roads for at least 10 minutes before testing. The warm-up was always done in subject-blinded Specialized Torch 
3.0 shoes (Specialized Bicycle Components Inc., Morgan Hill, CA, U.S.A.). Prior to the warmup, we adjusted the saddle height to each subject's preference. After the warm-up, subjects performed three practice rides of the sprint course and a recovery loop. We instructed the subjects to ride up to the sprint starting line at $20 \mathrm{~km} / \mathrm{hr}$ using visual feedback from the digital display of the Garmin 1000 and then sprint at maximal effort for $50 \mathrm{~m}$. Of note: the Garmin 1000 only displayed velocity, time, and distance ridden to the riders, not power output. The three practice rides allowed the subjects to become comfortable with the testing bicycle, the sprint course and the recovery loop, and they chose the specific gearing that they would use for the remainder of the experimental trials.

After the warm-up period, the subjects performed three experimental trials in each of the three different cycling shoe conditions for a total of nine trials. Using a difference of means paired t-test, we analysed a dataset provided by Hoogkamer, DeBortoli, and Kram (personal communication) for a protocol that used the same number of total trials and rest periods and determined that there were no statistical differences between the average power $(\mathrm{p}=0.92)$ or peak power $(\mathrm{p}=0.99)$ between the first trial versus the last trial. That indicates that fatigability was negligible with this experimental design.

In the present study, the shoes all comprised Specialized Torch $3.0 ®$ uppers with two $B O A ®$ dials and a lower Velcro strap. The shoe uppers were attached to three different commercially available cycling shoe soles: the Torch 1.0, the Torch 3.0, and the S-Works 7, all manufactured by Specialized. The Torch 1.0, the least stiff of the three soles, is made of injection-moulded nylon composite material and reported by Specialized to have a 6.0 stiffness index (Torch 1.0 Road Shoes, 2020). The Torch 3.0, the moderately stiff sole, was made of a carbon fibre - fibreglass composite blend with a reported stiffness index of 8.5 (Torch 3.0 Road 
Shoes, 2020). The S-Works 7, considered the stiffest of the three soles, was made of carbon fibre and reported to have a stiffness index of 15.0 (S-Works 7 Road Shoes, 2020). Specialized's method of stiffness indexing of shoes is proprietary. The cleat positions were uniformly set for all of the pairs of shoes by a Retul®-certified bike fitter (Retul, Boulder, CO, U.S.A.). The shoes were set in a neutral medio-lateral position, and with the cleat as far posterior as possible. Only one subject requested a different cleat position than the standardized cleat position and the cleats were toed medially inwards for that subject to help alleviate an uncomfortable knee angle. Two pairs of each model of cycling shoes were used for testing - one pair of EU size 43 and one pair of size 44. We blinded the shoe model to the subjects (single-blind) by matching the uppers of the cycling shoes and covering all of the graphics/logos.

Each subject rode each shoe condition three times in a quasi-mirrored design. The simplest mirrored design for two conditions, A and B, would have trial orders of either A,B,B,A or B,A,A,B. Ideally, a mirrored design compensates for learning or fatigue effects caused by the repeated sprinting. With three shoe conditions and three replicates of each condition, there are 24 possible orders of quasi-mirrored trial conditions. Table 1 shows the possible orders with each of the three shoes denoted as A, B and C. We randomly assigned each subject an order between 1 to 24 using an internet-based random number generator.

During the experimental trials, we required that each rider consistently used their own self-selected gear combination. The subjects rode with their hands in whatever position they desired, but we required them to keep it consistent across all trials. Twelve of the subjects rode in the handlebar drops and seven of the subjects rode with their hands on the brake hoods. Most subjects remained seated prior to the sprint and all subjects self-selected to stand during the sprint. 
During each trial, we recorded the ambient temperature and wind speed using a hand-held hotwire anemometer (Extech Hot Wire Thermo-Anemometer Model 407123: Waltham, MA, U.S.A.). Temperature varied on average $1.9 \pm 1.7$ degrees Celsius from beginning to end of each testing period. Testing was paused whenever the wind reading exceeded $3.0 \mathrm{~m} / \mathrm{s}$, and the maximum wind velocity averaged across all trials of the 19 participants was only $0.7 \pm 0.4 \mathrm{~m} / \mathrm{s}$ with the maximum individual wind reading recorded during any of the trials being $2.4 \mathrm{~m} / \mathrm{s}$. Of note: $0.7 \mathrm{~m} / \mathrm{s}$ is considered "light air" and our maximum wind reading of $2.4 \mathrm{~m} / \mathrm{s}$ is considered a "light breeze" by the Beaufort Wind Scale (Beaufort Wind Scale, 2016). We were not able to quantify wind direction.

After completion of a trial, subjects recovered for a minimum of 5-minutes before beginning another trial. The recovery period allowed the researchers to change the shoes of the subject, record subjective feedback, and verify the correct gear ratio. We instructed the subjects to maintain the same level of tightness in the upper of the shoes when they switched shoes between trials. Moreover, the recovery period limited the effects of fatigue caused by the repeated sprinting. Subjects completed all of the nine trials in a single experimental session that lasted approximately two hours.

The Quarq power meter sampled and transmitted data to the Garmin 1000 for storage. The Quarq power meter samples at $64 \mathrm{~Hz}$ internally but sends average data to the Garmin at 1 Hz. Independently, we used a Garmin hub-based velocity sensor and crank-based cadence sensor to record velocity and cadence data. We imported the raw data from the Garmin 1000 using Golden Cheetah analytic software (www.goldencheetah.org). Then, we analysed each of the nine sprints using a Microsoft Excel macro to determine the cadence, velocity, peak and 1 second average power, acceleration, and the work done during each of the nine $50 \mathrm{~m}$ sprints. 
Using R Studio, we used repeated measures ANOVAs to test for any main effects of sole type on each of the investigated performance parameters. We analysed the following variables: $50 \mathrm{~m}$ average and peak 1-second power, average and peak change in velocity, average and peak cadence, maximum sprint velocity, peak acceleration, and peak torque during the sprint. Additionally, we ran three repeated measures ANOVAs comparing maximal sprint velocities between trials 1 and $2(p=0.34), 2$ and $3(p=0.91)$, and 1 and $3(p=0.31)$ for all three shoe soles which showed there were no net learning/fatigue effects. Riders were not able maintain an approach speed of exactly $20.0 \mathrm{~km} / \mathrm{hr}$. To evaluate the effect of rider inconsistency in pre-sprint initiation velocity, we ran a repeated measures ANOVA which revealed that the subjects were not initiating sprints from consistently different starting velocities in the three different shoes $(\mathrm{p}$ $=0.89$ ). Lastly, the Pearson's product moment correlation coefficient between the pre-sprint initial velocity and the maximal sprint velocity was weak $r^{2}=0.034$ and not significant $(p=$ $0.66)$.

For each sprint trial, we plotted 1-second average power and velocity versus time to identify the beginning of the sprint trial. Figure 1 depicts a representative trial. The 1-second average power and velocity show that the sprint was initiated at $6 \mathrm{~s}$ and continued until $12 \mathrm{~s}$. In this example, 50 $\mathrm{m}$ average power was calculated between 6 and $12 \mathrm{~s}$ and the peak 1-second power occurred at 10 s. We calculated the average change in velocity as the average velocity during the sprint minus the pre-sprint initial velocity. This metric determined if subjects were maintaining their velocity better throughout the duration of the sprint. We calculated peak change in velocity as the difference between the peak velocity and the pre-sprint initial velocity. We calculated average cadence in RPM through the duration of the sprint. Peak cadence was the fastest cadence reached during the sprint. Maximal sprint velocity was the greatest 1 -second average peak 
velocity during the sprint. Due to the low time resolution of the data, only the 1 -second peak acceleration during the sprint trial is reported. We used the equation: torque $(\mathrm{N}-\mathrm{m})=$ power $(\mathrm{W})$ / $($ cadence $(\mathrm{RPM}) \cdot(\pi / 30))$ to calculate the 1 -second average peak torque.

We set significance at $p<0.05$ for all of the repeated measures ANOVAs. All values are reported as means \pm standard deviation. If we detected a main effect, we performed follow-up paired t-tests with a Bonferroni corrected $\mathrm{p}<0.017$ value $(0.05 / 3$ comparisons $)$.

\section{Results}

As shown in Table 2, none of the nine performance parameters investigated were significantly different across the three shoe conditions, $(\mathrm{p}>0.31)$. Graphic representations of the $50 \mathrm{~m}$ average and peak 1-second power for each subject are shown in Figures 2 and 3. Because none of the RMANOVAs detected any main effects, follow-up paired t-tests were not warranted.

\section{Discussion}

This study expands upon the findings of Burns and Kram (in press), who found greatly enhanced uphill sprint performance metrics with cycling shoes and clipless pedals versus flexible running shoes with toe-clip pedals and flat pedals. We investigated sprint performance metrics across a spectrum of commercially available road cycling shoes while controlling for shoe-pedal interface. We compared three road cycling shoe soles with different longitudinal bending stiffnesses but identical uppers. We found that there were no statistically significant differences between shoe conditions for: $50 \mathrm{~m}$ average or peak 1-second power, average or peak change in velocity, average or peak cadence, maximum sprint velocity, peak acceleration, or peak torque. We therefore reject our hypothesis that the longitudinally stiffest shoes would yield the greatest 
performance during short uphill sprints. We retain the null hypothesis that there would be no performance differences between the three different cycling shoe soles.

Overall, we found that increased longitudinal bending stiffness of the cycling shoe sole has no effect on performance metrics during sprint cycling at least in recreational/competitive cyclists. Our findings are consistent with Koch et al. (2013) who compared sprint cycling with sham versus carbon fibre insoles and found no performance benefits from the increased stiffness. It is not yet clear what sole stiffness is optimal. Somewhat less stiff cycling shoe soles may help to prevent or alleviate metatarsalgia or ischemia that can occur in cyclists due to the increased plantar pressures associated with carbon fibre cycling shoe soles (Jarboe and Quesada, 2003). Perhaps designers of cycling shoes should focus on aspects of shoe design such as closure and comfort rather than striving to increase sole stiffness.

Since our results may be counterintuitive, we estimated the mechanical energy that would be dissipated by a cycling shoe sole based on previous measurements of sole stiffness (Jarboe and Quesada, 2003). In cycling, the resultant force vector applied by the leg and foot passes very near the pedal spindle and thus it is difficult to apply a large bending moment to the shoe sole. In the worst case, a force applied at the most posterior point on the sole (the heel) would create the largest bending moment. In fact, this is similar to how Jarboe and Quesada (2003) tested their cycling shoes. They applied a force (F) of $250 \mathrm{~N}, 0.102 \mathrm{~m}$ posterior from a cleat fixture. They measured the longitudinal bending stiffness ( $\mathrm{k}$ ) of carbon fibre composite and homogenous plastic cycling shoe soles as $10,070 \mathrm{~N} / \mathrm{m}$ and $7,790 \mathrm{~N} / \mathrm{m}$, respectively. If the shoe sole and its displacement $(\Delta \mathrm{x})$ are modelled as a simple linear Hookean spring, the equation $0.5 \cdot \mathrm{k} \cdot \Delta \mathrm{x}^{2}$ can be used to calculate the mechanical energy temporarily stored in the cycling shoe sole. Using Hooke's law $(\mathrm{F}=\mathrm{k} \cdot \Delta \mathrm{x})$, a $100 \mathrm{~N}$ force applied at the heel of the carbon fibre composite shoe 
would cause almost exactly a $1 \mathrm{~cm}$ displacement $(0.00993 \mathrm{~m})$ and would store $0.504 \mathrm{~J}$ of energy per shoe per pedal cycle. For the homogeneous plastic sole, a $100 \mathrm{~N}$ force would cause a greater displacement $(0.013 \mathrm{~m}, \sim 1.3 \mathrm{~cm})$ and would store $0.658 \mathrm{~J}$ per shoe per pedal cycle.

Unlike in running, there is no evidence that a shoe can usefully store and return elastic energy during cycling. Thus, we assumed that any mechanical energy used to deform a cycling shoe sole during the downstroke is lost during the upstroke. During a sprint at a cadence of 120 RPM, $0.504 \mathrm{~J}$ per shoe per pedal cycle equates to almost exactly $2 \mathrm{~W}(1 \mathrm{~W}$ per shoe) of power dissipated for the carbon fibre composite sole versus $2.6 \mathrm{~W}$ dissipated for the homogeneous plastic sole. As we measured in this study, many riders produce $1000 \mathrm{~W}$ during a sprint. Under the worst case assumptions above, a $0.6 \mathrm{~W}$ difference in dissipation between shoe sole types is $<$ $0.1 \%$. Given these calculations, our findings of no differences in performance metrics during maximal sprinting between shoe sole types make sense.

In Figure 3, we depict the combined findings of Burns and Kram (in press) and the present study. We have shown there to be no significant differences in performance between our three shoe sole conditions but Burns and Kram found up to $27.6 \%$ differences in sprint performance metrics between cycling shoes with clipless pedals and flexible running shoes with two different pedal conditions. However, neither study identified a critical sole stiffness below which performance is impaired. Identifying that stiffness could facilitate optimizing sole stiffness for both performance and comfort.

Given the differing materials used in each of the three shoes, it is reasonable to consider the nylon composite Torch 1.0 soles to be the least stiff, the carbon fibre - fibreglass composite Torch 3.0 soles to be medium stiffness, and the carbon fibre sole of the S-Works 7 to be the most stiff. However, it would be useful to develop a standardized, public domain test protocol for 
cycling shoe sole stiffness so that companies and researchers could report stiffness in consistent SI units similar to the (now recalled) ASTM F911 used for testing the flexibility of running shoes.

We recognize that this study has its limitations. The Quarq power meter samples torque at $64 \mathrm{~Hz}$, but only reports averages to the Garmin 1000 at $1 \mathrm{~Hz}$. The low sampling frequency may have prevented us from detecting a small difference between the three cycling shoes in term of instantaneous power. Additionally, the study results are specific to $50 \mathrm{~m}$ sprints up a $4.9 \%$ grade. We made no attempt to examine flat, steeper, or longer sprints. It is possible that greater sole stiffness may have an effect during longer sprints as a rider fatigues. All of the 19 participants were male riders but we would not expect different results for female riders. Due to their greater power outputs, it is possible that elite sprinters might realize small gains from the use of stiffer cycling shoe soles as compared to recreational/competitive cyclists.

In conclusion, we found no difference in performance between less stiff and stiffer road cycling shoe soles during short uphill sprints in recreational/competitive cyclists. The stiffest cycling shoe soles showed no performance benefits in: $50 \mathrm{~m}$ average and peak 1-second power, average and peak change in velocity, maximum velocity, peak acceleration, or peak torque compared to a moderately stiff and the least stiff road cycling shoe soles offered by a wellknown manufacturer.

\section{Acknowledgements}

The authors would like to thank Todd Carver for help facilitating the study and useful comments on an earlier draft. Additional thanks go to Dr. Wouter Hoogkamer for sharing his expertise and preliminary data from an earlier study. 


\section{Disclosure Statement}

Specialized Bicycle Components Inc. financially supports bicycle research in the Locomotion Laboratory but did not commission or fund this specific study. 


\section{References}

1. Anderson, J., \& Sockler, J. (1990). Effects of orthoses on selected physiologic parameters in cycling. Journal of the American Podiatric Medical Association, 80(3), 161-166. doi: $10.7547 / 87507315-80-3-161$

2. Beaufort Wind Scale. (2016, September 2). Retrieved February 27, 2020, from https://www.weather.gov/mfl/beaufort

3. Burns, A.C. \& Kram, R. (2019). The effect of cycling shoes and the shoe-pedal interface on maximal mechanical power output in bicycling, SportRxiv. doi:10.31236/osf.io/wkxsz.

4. Fizik R5B Uomo Men's road shoes. (2015, October 15). Retrieved February 27, 2020, from https://road.cc/content/review/146066-fizik-r5b-uomo-mens-road-shoes

5. Infinito R1. (n.d.). Retrieved February 27, 2020, from https://www.fizik.com/us_en/infinitor1.html\#.

6. Jarboe, N. E., \& Quesada, P. M. (2003). The effects of cycling shoe stiffness on forefoot pressure. Foot \& Ankle International, 24(10), 784-788. doi: 10.1177/107110070302401009

7. Koch, M., Frohlich, M., Emrich, E., \& Urhausen, A. (2013). The impact of carbon insoles in cycling on performance in the Wingate anaerobic test. Journal of Science and Cycling, 2(2).

8. Mornieux, G., Stapelfeldt, B., Gollhofer, A., \& Belli, A. (2008). Effects of pedal type and pull-up action during cycling. International Journal of Sports Medicine, 29(10), 817-822. doi: $10.1055 / \mathrm{s}-2008-1038374$

9. Ostler, L. M., Betts, J. A., \& Gore, C. J. (2008). Gross cycling efficiency is not altered with and without toe-clips. Journal of Sports Sciences, 26(1), 47-55. doi: $10.1080 / 02640410701332507$ 
10. RC9. (n.d.). Retrieved February 27, 2020, from https://bike.shimano.com/enUS/product/apparel-accessories/s-phyre/SH-RC901.html

11. Solestar BLK. (n.d.). Retrieved February 27, 2020, from https://www.solestar.de/en/solestarkontrol-blk.html

12. S-Works 7 Road Shoes. (n.d.). Retrieved February 27, 2020, from https://www.specialized.com/us/en/s-works-7-road-shoes/p/157321?color=232549-157321

13. S-Works EXOS Road Shoes. (n.d.). Retrieved February 27, 2020, from https://www.specialized.com/us/en/s-works-exos-road-shoes/p/157750?color=237037157750

14. Straw, A. H., \& Kram, R. (2016). Effects of shoe type and shoe-pedal interface on the metabolic cost of bicycling. Footwear Science, 8(1), 19-22. doi: $10.1080 / 19424280.2016 .1140817$

15. Torch 1.0 Road Shoes. (n.d.). Retrieved February 27, 2020, from https://www.specialized.com/us/en/torch-1-0-road-shoes/p/172702?color=272400-172702

16. Torch 3.0 Road Shoes. (n.d.). Retrieved February 27, 2020, from https://www.specialized.com/us/en/torch-3-0-road-shoes/p/155410?color=237052-155410 
Tables:

\begin{tabular}{|c|c|}
\hline Number & Testing Order \\
\hline 1 & A B C B A C B C A \\
\hline 3 & A B C B A C C B A \\
\hline 4 & A B C C A B B C A \\
\hline 5 & A B C C A B C B A \\
\hline 6 & A C B B A C B C A \\
\hline 7 & A C B B A C C B A \\
\hline 8 & A C B C A B B C A \\
\hline 9 & A C B C A B C B A \\
\hline 10 & B C A A B C A C B \\
\hline 11 & B C A A B C C A B \\
\hline 12 & B C A C B A A C B \\
\hline 13 & B C A C B A C A B \\
\hline 14 & B A C A B C A C B \\
\hline 15 & B A C A B C C A B \\
\hline 16 & B A C C B A A C B \\
\hline 17 & B A C C B A C A B \\
\hline 18 & C A B A C B A B C \\
\hline 19 & C A B A C B B A C \\
\hline 20 & C A B B C A A B C \\
\hline 21 & C A B B C A B A C \\
\hline 23 & C B A C B A B C \\
\hline 24 & C B A B C A A B C \\
\hline & C B A B C A B A C \\
\hline
\end{tabular}

Table 1: 24 possible orders of trials based upon three conditions (A, B, and C) with three replicates. 


\begin{tabular}{|c|c|c|c|c|}
\hline & Torch 1.0 & Torch 3.0 & S-Works 7 & $\begin{array}{c}\text { RMANOVA P- } \\
\text { Value }\end{array}$ \\
\hline $\begin{array}{c}\text { 50 m Average } \\
\text { Power (W) }\end{array}$ & $880.3 \pm 139.5$ & $874.1 \pm 159.9$ & $874.3 \pm 139.2$ & 0.76 \\
\hline $\begin{array}{c}\text { Peak 1-Second } \\
\text { Power (W) }\end{array}$ & $1056.1 \pm 173.3$ & $1057.1 \pm 181.1$ & $1046.5 \pm 162.9$ & 0.39 \\
\hline $\begin{array}{c}\text { Average Change in } \\
\text { Velocity (m/s) }\end{array}$ & $2.31 \pm 0.54$ & $2.38 \pm 0.55$ & $2.34 \pm 0.46$ & 0.31 \\
\hline $\begin{array}{c}\text { Peak Change in } \\
\text { Velocity (m/s) }\end{array}$ & $3.91 \pm 0.98$ & $3.91 \pm 0.97$ & $3.85 \pm 0.85$ & 0.66 \\
\hline $\begin{array}{c}\text { Average Cadence } \\
\text { (RPM) }\end{array}$ & $93.4 \pm 9.9$ & $93.8 \pm 10.0$ & $93.0 \pm 9.5$ & 0.67 \\
\hline $\begin{array}{c}\text { Peak Cadence } \\
\text { (RPM) }\end{array}$ & $104.7 \pm 12.0$ & $104.7 \pm 11.7$ & $104.3 \pm 11.4$ & 0.81 \\
\hline $\begin{array}{c}\text { Maximum Sprint } \\
\text { Velocity (m/s) }\end{array}$ & $9.80 \pm 1.09$ & $9.78 \pm 0.97$ & $9.75 \pm 0.84$ & 0.89 \\
\hline $\begin{array}{c}\text { Peak Acceleration } \\
\text { (m/s } \mathbf{2})\end{array}$ & $1.34 \pm 0.29$ & $1.35 \pm 0.29$ & $1.32 \pm 0.23$ & 0.76 \\
\hline $\begin{array}{c}\text { Peak Torque } \\
\text { (N-m) }\end{array}$ & $105.1 \pm 14.3$ & $104.8 \pm 15.3$ & $105.0 \pm 15.1$ & 0.95 \\
\hline
\end{tabular}

Table 2: None of these performance variables showed any significant main effects for sole stiffness during a $50 \mathrm{~m}$ uphill sprint $(\mathrm{n}=19)$. 
Figures: 


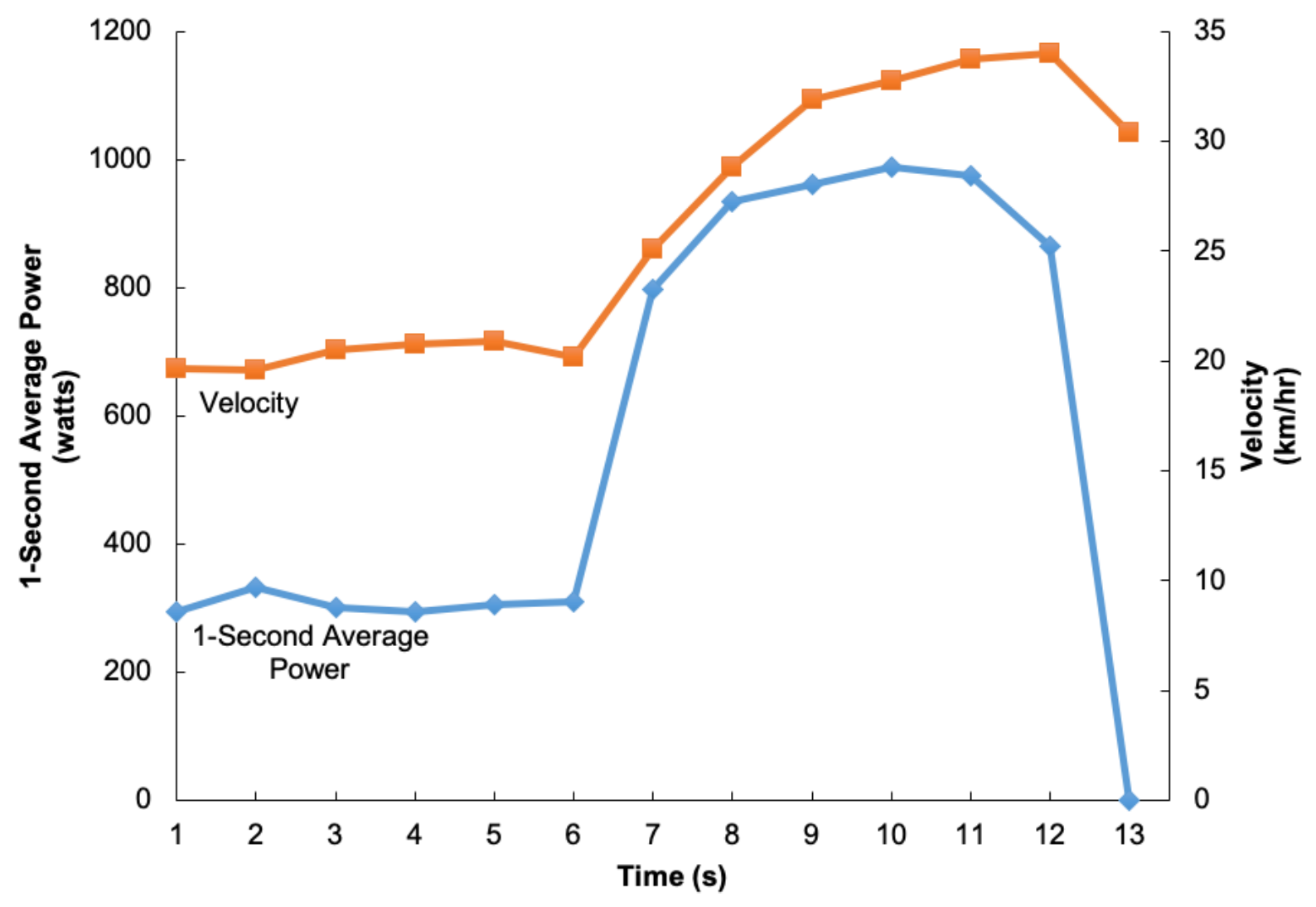



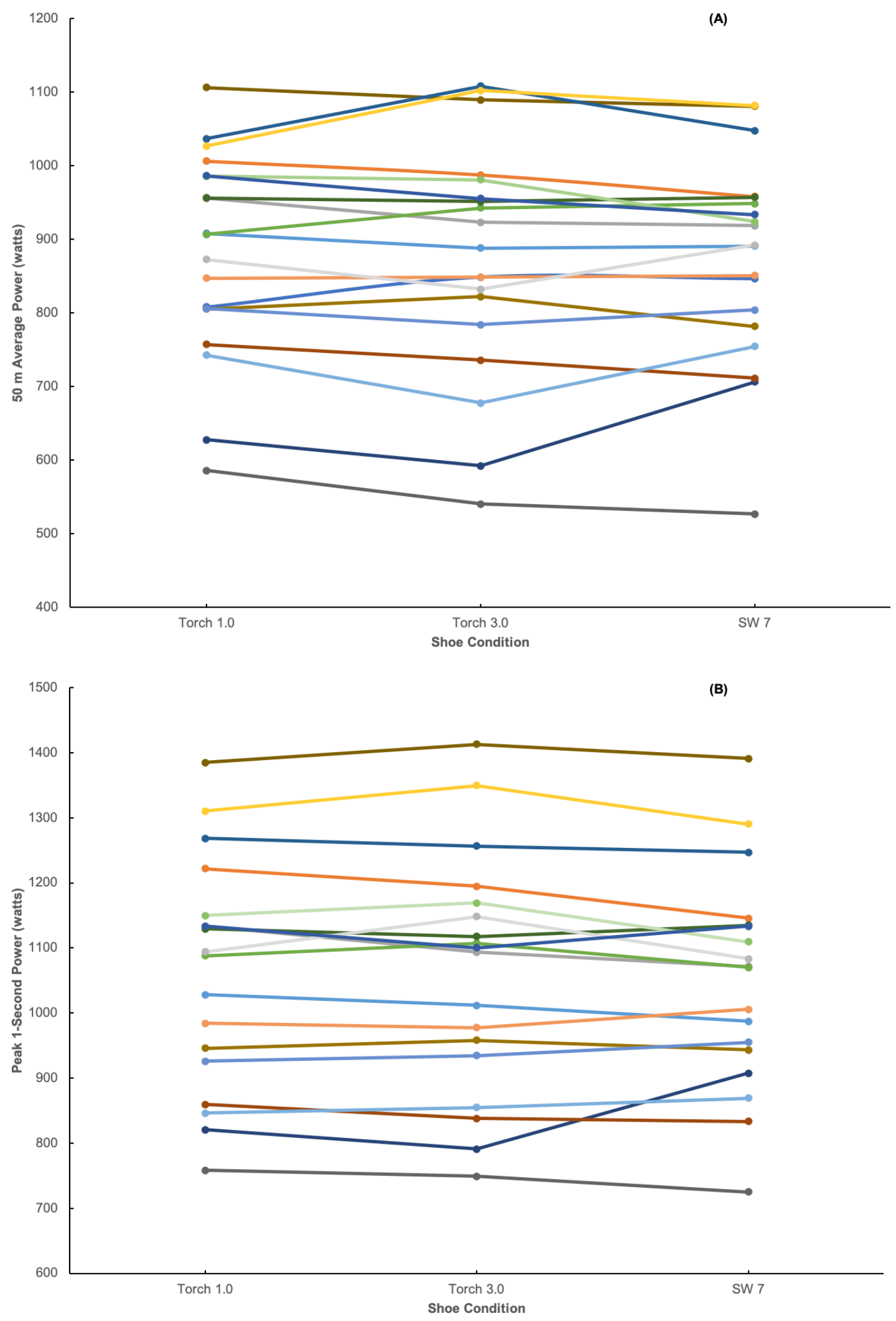


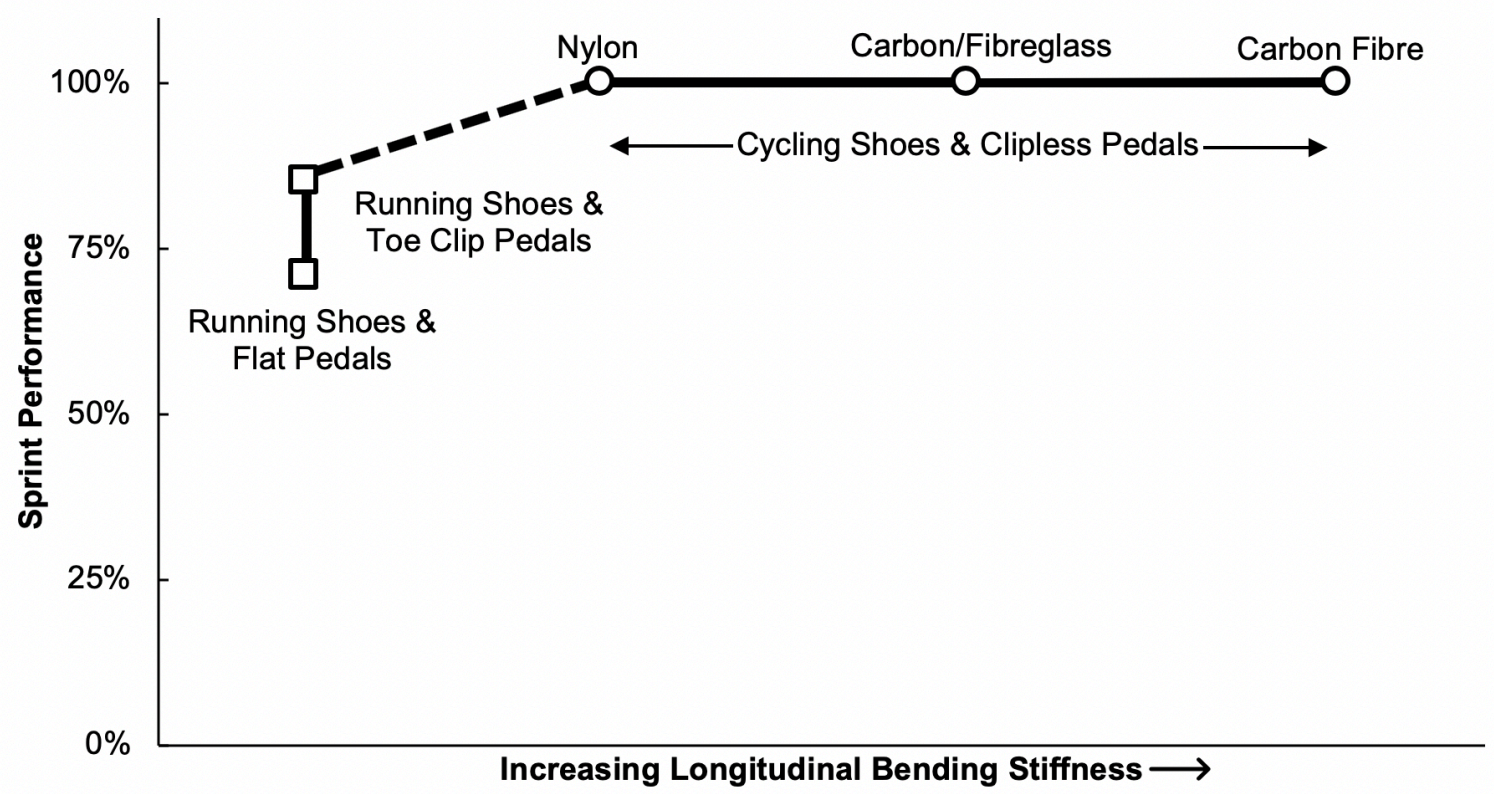


Figure 1: Plots of 1-second average power and velocity versus time for a representative sprint trial.

Figure 2A: $50 \mathrm{~m}$ average power versus shoe condition. 2B: Peak 1-second power versus shoe condition. Each of the 19 subjects is represented by a line.

Figure 3: Schematic graph of sprint performance versus longitudinal bending stiffness (not to scale). Solid lines and symbols are for empirically measured results presented here and Burns and Kram (in press).

Dashed line for relation yet to be determined. 